\title{
General Discussion to the Papers of Dr Keane, Dr Maury, Dr Berard, Dr A. Heilporn and Dr H. J. Hachen
}

Dr L. Michaelis (G.B.). There is just one point the reason for which I get up. In the whole discussion I have not heard anything about prevention of pain. I think it is a generalisation which will be accepted that the smaller the number of patients with severe chronic pain the better the quality of all-round treatment and if, as Dr Hachen's paper has shown, there is a comparatively large number with intense chronic pain there is generally a history of what, on the whole, we now consider faulty treatment. There were, if I remember rightly, some 60 per cent of his patients who had laminectomies and that because it produces meningitis and is followed by adhesions in the meninges and that is one of the main causes of pain. The second and repeatedly proved cause of intense chronic pain is myelography. The myelography, except that done with air, is a definite cause of meningitic adhesions and are a definite cause of chronic severe pain. It is understood that the quality of treatment, prevention of contractures, the treatment of the spasticity therefore must be good or you will get pain, but let us not forget that we all used to see patients, I don't think they are as frequent now, who complained about severe pain without us being able to find any localised cause for it, simply because they were so ill with the toxicity from their pressure sores or their intense urinary infection. In these cases, proper treatment both by antibiotics and, even more important, by blood transfusions was all that was needed to reduce the pain either altogether or to a bearable level. I feel, with the trying problem of pain to the para- or tetraplegic, we must try on our side to do everything to prevent the known causes of pain in these patients.

DR SEGAL (U.S.A.). I'd like to ask Dr Hachen a question. You separated your patients after 7 days and you carried on for 3 months. Did you after 3 months survey, separately, those first two categories: those with little relief compared with those with moderate to good relief.

DR HACHEN. With regard to evaluation of the data, we can only give quantitive or semi-quantitive assessment after I week with precision because the stimulation parameters were identical in all the patients and treatment was under regular supervision of the medical team, but later on many patients continued with home stimulation and especially what happened between the 2nd and 3rd month. We are quite sure what happens with drug treatment that some patients must not have adhered strictly to our prescription, and that there was irregularity as to length of duration and quite a lot of variation in stimulation parameters so that we get an over-all impression but nothing measurable quantitatively.

Dr Segal (U.S.A.). The reason I was interested in asking this question, did most of the failures come from the poor results after I week. Would you be able to ascertain that?

Dr Hachen. No. Some patients even improved, they had better results after 2 weeks than after I week and this may be in relation to difficulty of locating the optimal trigger zones. People have recently performed acupuncture previously to transcutaneous low intensive stimulation and it may be that optimal results are obtained by stimulating the best acupuncture sites, so that I think there is a close relation between acupuncture activity and transcutaneous electric stimulation, both acting as actual counter-irritant techniques.

SIR Ludwig GutTmann (G.B.). It is a well known fact that any stimulation in any part of the body can counteract existing pain. I have had some personal experience when I broke my own spine in Egypt some years ago. I had agonising pain in my back when I was taken to the house of a colleague. There was a nurse there and I asked her to give me something against the pain but not morphia. She had some novalgine and injected this in my right thigh underneath the periost. The pain in my back disappeared at once because the pain in my thigh was so overwhelming. I agree with Michaelis it was our principle in Stoke Mandeville in treating patients with pain in the acute stage as well as in the sub-acute stage by concentration through work. In the War, I got patients who were treated with morphia and other drugs and when they arrived at the Unit the first question 
some of them asked was 'When can I get the next jab?' I think most of those patients are still waiting for that. There is no question that in the early treatment of paraplegics the treatment by concentration through work and sport has proved to have a fascinating effect. I want now to discuss two or three methods which have been introduced for relieving pain. Maury and other colleagues have emphasised the importance of the psychological state of the patient which has to be examined by the doctor who wants to relieve pain extremely carefully from every point of view. In cauda equina or incomplete low distal cord lesions we have to consider here very carefully whether the person is impotent or not. I was asked to see a man with an incomplete cauda-equina lesion with pain, both the burning pain and the spasmodic radicular pain. My first question was about the nature of the pain and whether the pain was constant. 'Yes the burning pain is constant but the other comes on particularly when I'm tired or have some problems.' The next question was 'What about your sexual function?' He said 'Oh that's very good'. He asked me for my views about a cordotomy which was suggested to him. I explained that unilateral cordotomy is of no use but bilateral cordotomy would have the danger of resulting in impotence and that he had to consider. 'Oh thank you, I prefer my potency'. This is a point which can have legal implications, if the surgeon who advises bilateral cordotomy in such a case does not inform the patient properly. The other problem is the paramedullary or radicular injections with pain-killing substances. Greatest care should be taken in attempting paravertebral and in particular epidural injections for relieving pain especially in the upper thoracic level ( $\mathrm{T}_{3}$ to $\mathrm{T} 6$ ), as paraplegia can result which may lead to legal procedures.

The third point is the intrathecal injection of alcohol which I introduced in paraplegics in 1945 in cases with complete lesions or extremely severe lesions with intractable spasticity and pain and where I described indications and contra-indications. The same should apply to the injections of phenol which have been recommended for pain relief of individual spinal root pain selectively in patients with cancer. We have received patients at Stoke Mandeville who had more or less severe paralysis of the bladder afterwards. It was mentioned in early publications on phenol that this complication is only temporary, but the fact remains that in some cases the paralysis of the bladder was permanent. Therefore as much as we demand now that when in the immediate stage of spinal cord injuries a neurosurgeon or orthopaedic surgeon decides to do a laminectomy or fusion, he has to inform the patient and also in particular his family, properly, as otherwise he might get into trouble: the same applies to para-spinal and intrathecal injections for pain.

Finally, with regard to laminectomy to relieve pain in later stages of spinal cord injuries, I mention a soldier who sustained a gunshot injury at $\mathrm{T}_{7}$ and he was complaining continuously about the root pain at the level of the lesion and although he was somewhat of a psychological personality, I decided 4 months after the injury to operate. After opening the dura I found that the left posterior root of $\mathrm{C}_{7}$ was pulled to the other side and was continucusly stretched. I divided the root as well as the adhesions at the level of injury and the man was relieved from pain for many weeks completely, but then the pain returned although less marked but improved considerably once his pension case was settled and he had no worries any more.

Dr Pinkerton (Canada). I was interested in Dr Berard's paper on testicular pain but he didn't comment upon whether any of the patients complained of spontaneous testicular pain. This is something I have noted in some patients who either have anal pain which radiates to the testicles or testicular pain which tends to radiate to the anal area, which is most disturbing. One patient in particular at the present moment, who has a history of previous tuberculosis of the testicle 4 years before his cervical injury. He has some crude sacral sparing but otherwise is a complete lesion at C6/7 and in regard to the transcutaneous nerve stimulation perhaps Dr Hachen would comment upon treating testicular pain in this way, and whether the placement of the electrodes would be at Tio or where he might place them and the frequency of stimulation in that type of situation.

DR BERARD. I didn't speak about spontaneous pain in the testes but only of produced pain by squeezing and its semiological value, of course.

DR PINKERTON. I realise that. 
DR BERARD. On the other hand you can see for instance in orchitis, on the one side there is a level of Tio on the other side there is a level of Tir. The patient feels the orchitis on the side of TII and not on the other side, and we saw this phenomena several times, three or four times, but the problem of pain in the testes in cervical spine injuries, I'm not able to explain that.

DR HACHEN. When we selected these patients we made three major rules, so as to have some sort of homogeneity in these patients we took only pain at the fracture site, pain of particular origin and true phantom pain. We have actually no cases involved of pain in the renal area. I would assume that we would have to experiment and see what parameters would be most essential. It is very hard to predict that one can obtain any effect. As I showed in this series, phantom pain usually responds poorly to transcutaneous stimulation and the best results, like in Heilporn's paper. This seems to prove the identity of the results that the best results are obtained in a dorsal root lesion. Phantom limb is a poor indication for transcutaneous stimulation.

Dr HANSA (Egypt). I have tried transcutaneous stimulation in some paraplegic patients and then I found that most patients who are complaining of phantom pain or get pain are those who are lower lesions $\mathrm{LI}_{\mathrm{I}}$ and $\mathrm{T}_{\mathrm{I}} 2$ and specially incomplete transections of the cord. I tried transcutaneous stimulation and I agree with Dr Hachen and with his results that about 50 per cent are due to psychological factors as Sir Ludwig Guttmann said, and second due to placebo reaction. I tried this while the machine was switched off and the patient said to me, 'Oh it's marvellous'.

DR MENTER (U.S.A.). One of the unfortunate facts on the American scene of spinal injuries is that we have a large number of gunshot wounds. It's been my clinical impression, I think our hospital experience is, that these particular individuals even when referred early have significantly different pain patterns. Does anyone have any speculation as to why we have had no specific therapeutic programme that has been beneficial other than time, patience and reassurance, and many of these people do get better but they seem to have a definitely higher residual of hyperaesthetic sensations? Does anyone have a comment on that or an answer?

Dr SILver (G.B.). I'd like to ask some questions of Dr Hachen. He gave some contra-indications to the transcutaneous stimulation. Has he ever seen the pain made worse by this treatment and are there any other reasons for not carrying out this treatment, or any complications from the drug therapy regime? And a question to any of the speakers to add to the ones that have just been placed, it seems to me that the pain in the anus and the testicles is a very common one if you question the patients. Is there any reason why the pain seems to appear some months after injury? It's uncommon to come across these severe burning pains within days or weeks of injury; it appears to come on after some 2 or 3 months.

DR HACHEN. I'd just first comment to what my colleague from Egypt said. I totally agree with him that there must be a very heavy placebo effect in this technique, there is no doubt about it, but this may account partly for the difference between the early I-week results and the late 3-month results, because it is hard to accept that the placebo effect will persist for such a long time, placebo effects usually disappear within a short period of application of a new device. Secondly, to Dr Silver, we have had no other contra-indications observed. In patients with incomplete lesions one has to use various levels of stimulations which means a frequency of intensity of stimulus that provides paraesthesia and sensation of heat. These are the optimal stimulation parameters, if one uses frequency intensities that are too low one cannot expect any improvement.

DR Dollfus. One question from the chair: Has anybody got any experience of pain in paraplegic children? Because I have noticed that with paraplegic children, at least at Stoke Mandeville, very few of them complain of pain, and there might be a key there.

Mr Harris (G.B.). I wonder if I could ask Dr Berard about the syndrome mentioned, posterior cord syndrome. Testicular sensibility was not present in this syndrome. The second point is that unfortunately our attempts at helping pain with transcutaneous stimulation especially pain from arachnoiditis-I may say that this is not at all due to 
myelography-have been very disappointing. We've had no satisfaction in our patients at all.

DR BERARD. First of all posterior cord syndromes, I mean posterior compression for instance due to ependymoma. When both posterior tracts are compressed there is always an ataxia and testicular anaesthesia. Is that enough ?

Mr HARRIS. I'm sorry that is not quite enough.

SiR Ludwig Guttmann. I just wanted to answer Dr Menter of Colorado who raised an important question of pain increasing in gunshot injuries. From my war experience I would say the answer is no. Whether this was a different type of material (soldiers wounded in World War II) or whether this applies as you said to civilian cases it would be interesting to investigate.

I was interested in that paper of $\mathrm{Dr}$ Keane. He quite rightly shows concern of the hazards of lifting and turning cervical patients by hand, and I was pleased to see his findings, although it is well known how dangerous the turning by hand is, especially in fractures of the cervical spine. That was the reason why we introduced the Egerton-Stoke Mandeville turning and tilting bed to obviate the manual lifting and turning by three to four members of the nursing staff which was necessary in the method of postural reduction on pillows or sorbo rubber packs with regular turning every 2 hours day and night, which I introduced during World War II.
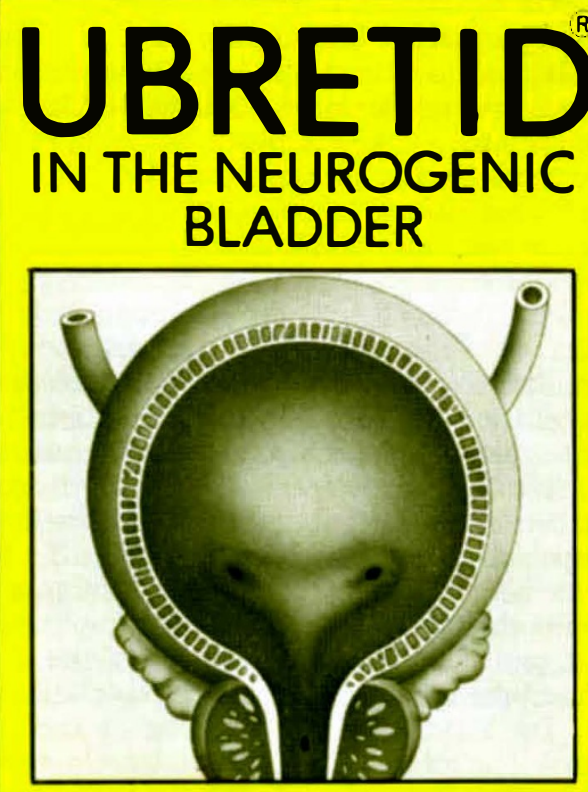

\section{'Where there was no} significant urethral or bladder outlet obstruction, Ubretid (distigmine bromide) produced a significant degree of bladder emptying in $58 \%$ of patients with an upper motor neurone neurogenic

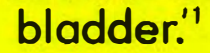

Ref 1: Yeo, J., Southwell, P., Rutowski, S., Marchant-Williams, H. (1974) Med. J.

Aust., 2, 201

Further information is available on request to: Berk Pharmaceuticals Limited Shalford, Surrey

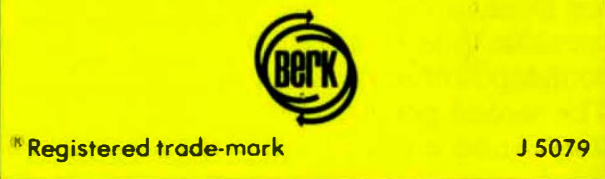

